\title{
Live attenuated enterovirus vaccine (OPV) is not associated with islet autoimmunity in children with genetic susceptibility to type 1 diabetes: prospective cohort study
}

\author{
Hanna Viskari ${ }^{1,2}$ - Sami Oikarinen ${ }^{1,3}$ - Sanna Hoppu ${ }^{4}$. Tytti Vuorinen ${ }^{5,6}$. \\ Heini Huhtala ${ }^{7}$.Jorma Toppari ${ }^{8,9} \cdot$ Riitta Veijola $^{10}$ - Jorma Ilonen ${ }^{11}$ • \\ Mikael Knip ${ }^{12,13,14,15,}$ • Heikki Hyöty ${ }^{1,3}$
}

Received: 23 May 2017 / Accepted: 10 July 2017 /Published online: 2 September 2017

(C) Springer-Verlag GmbH Germany 2017

\begin{abstract}
Aims/hypothesis Animal and human studies have implied that enterovirus infections may modulate the risk of islet autoimmunity and type 1 diabetes. We set out to assess whether serial administration of live oral poliovirus vaccine (OPV) in early life can influence the initiation of islet autoimmunity in a cohort of genetically predisposed children.

Methods OPV was administered to 64 children and a further 251 children received inactivated poliovirus vaccine (IPV). The emergence of type 1 diabetes-associated autoantibodies in serum (autoantibodies to GAD, insulinoma-associated protein 2 , insulin and islet cells) was monitored during prospective follow-up. Stool and serum samples were collected for enterovirus detection by RT-PCR.
\end{abstract}

Electronic supplementary material The online version of this article (doi:10.1007/s00125-017-4410-4) contains peer-reviewed but unedited supplementary material, which is available to authorised users.

Hanna Viskari

hanna.viskari@uta.fi

1 Department of Virology, Faculty of Medicine and Life Sciences, University of Tampere, Tampere, Finland

2 Department of Internal Medicine, Tampere University Hospital, 33520 Tampere, Finland

3 Fimlab Laboratories, Pirkanmaa Hospital District, Tampere, Finland

4 Department of Intensive Care, Tampere University Hospital, Tampere, Finland

5 Department of Clinical Virology, Turku University Hospital, Turku, Finland

6 Department of Virology, University of Turku, Turku, Finland

7 Faculty of Social Sciences, University of Tampere, Tampere, Finland
Results Administration of OPV increased enterovirus detected in stool samples from $11.3 \%$ to $38.9 \%(p<0.001)$ during the first year of life. During the follow-up (median 11.0 years), at least one autoantibody was detected in $17.2 \%$ of children vaccinated with OPV and $19.1 \%$ with IPV $(p=0.723)$. At least two autoantibodies were observed in $3.1 \%$ and $6.8 \%$ of children, respectively $(p=0.384)$.

Conclusions/interpretation Replication of attenuated poliovirus strains in gut mucosa is not associated with an increased risk of islet autoimmunity.

Trial registration ClinicalTrials.gov: NCT02961595

Keywords Autoantibody $\cdot$ Enterovirus $\cdot$ Inactivated polio vaccine $\cdot$ Oral polio vaccine $\cdot$ Type 1 diabetes

8 Department of Physiology, Institute of Biomedicine, University of Turku, Turku, Finland

9 Department of Pediatrics, Turku University Hospital, Turku, Finland

10 Department of Pediatrics, PEDEGO Research Unit, Medical Research Center, Oulu University Hospital and University of Oulu, Oulu, Finland

11 Immunogenetics Laboratory, University of Turku and Turku University Hospital, Turku, Finland

12 Children's Hospital, University of Helsinki and Helsinki University Hospital, Helsinki, Finland

13 Research Programs Unit, Diabetes and Obesity, University of Helsinki, Helsinki, Finland

14 Department of Pediatrics, Tampere University Hospital, Tampere, Finland

15 Folklhälsan Research Center, Helsinki, Finland 


$\begin{array}{ll}\text { Abbreviations } \\ \text { DIPP } & \text { Type 1 Diabetes Prediction and Prevention study } \\ \text { GADA } & \text { GAD autoantibodies } \\ \text { IA-2A } & \text { Insulinoma-associated protein } 2 \text { antibodies } \\ \text { IAA } & \text { Insulin autoantibodies } \\ \text { ICA } & \text { Islet cell antibodies } \\ \text { IPV } & \text { Inactivated polio vaccine } \\ \text { OPV } & \text { Live attenuated oral polio vaccine }\end{array}$

\section{Introduction}

Enteroviruses have been associated with type 1 diabetes in several studies $[1,2]$. These viruses have become increasingly interesting targets of potential preventive interventions in type 1 diabetes [3], and clinical trials with enterovirus vaccines would be needed to evaluate a possible causal relationship. Both live attenuated and inactivated enterovirus vaccines have long been used against polioviruses, the well-known examples of enteroviruses. Regarding other enteroviruses, vaccines against enterovirus 71 have passed phase III trials only recently [4].

The disease process leading to type 1 diabetes is usually slow. Disease-associated autoantibodies which target pancreatic islet autoantigens and predict the development of type 1 diabetes are the first detectable markers of the initiation of this process $[5,6]$. These autoantibodies often appear at a young age. In a recent report that combined data from three birth cohort studies from Colorado (USA), Germany and Finland, the median age at the appearance of multiple islet autoantibodies was only 2.1 years, while clinical diabetes was diagnosed at a median age of 6.1 years [5]. Genetic susceptibility to the disease is regulated mainly by the genes in the HLA locus but also by several non-HLA genes, most of which are involved in the regulation of the immune responses [7].

Environmental factors play an important role in the pathogenesis of type 1 diabetes and the most studied candidates include enteroviruses. These viruses are among the most common viruses to infect humans worldwide and include more than 100 genotypes (human enterovirus species A-D) [8, 9]. Infections have a seasonal pattern, especially in temperate countries such as Finland, peaking in the summer and autumn. Collectively, enteroviruses can infect almost all human tissues but various genotypes have specific tropism to certain organs and cells, which is partly regulated by the attachment of different viruses to different host cell receptors [8]. For example, polioviruses show neurotropism by attaching to specific poliovirus receptors in the anterior spinal cord. Furthermore, certain enterovirus types have tropism to human pancreatic islets causing islet damage and inflammation during infection [10]. Enterovirus protein has been detected in pancreatic islets in the majority of individuals with type 1 diabetes [11].

Poliovirus vaccines offer a useful model for the development of vaccines against other enteroviruses. Two types of poliovirus vaccines, the inactivated (IPV) and the live attenuated 'oral' (OPV) vaccines, have been used worldwide since the mid-twentieth century. IPV contains formalin-inactivated polioviruses and is administered by parenteral injection leading to immunisation, whereas OPV includes infective but attenuated viruses that are administered orally leading to subclinical infection in vaccine recipients. OPV is highly immunogenic inducing humoral and cell-mediated antiviral immune responses and mucosal immunity, while IPV induces mainly humoral immune responses. Both are generally safe but OPV may rarely cause prolonged infection or major adverse effects especially in immunocompromised recipients [9].

A possible association between poliovirus vaccinations and the risk of type 1 diabetes or development of islet autoantibodies has been analysed in a few studies [12-14]. However, these studies have not distinguished between IPV and OPV. Nevertheless, no indication of an increased risk of type 1 diabetes or islet autoimmunity has been seen in individuals vaccinated against polio. Since OPV induces a stronger immune response than IPV and inflammation in the gut mucosa, and might thus change the immunological milieu in the closely located pancreas and its lymphatic networks, we carried out a study to test whether children who are exposed to live attenuated poliovirus by serial OPV administration early in life have a distinct risk of developing diabetes-associated autoantibodies compared with children vaccinated with IPV.

\section{Methods}

Participants This study was carried out in a birth cohort of the ongoing prospective Type 1 Diabetes Prediction and Prevention (DIPP) study in Tampere, Finland, as previously described $[15,16]$. Briefly, all babies whose parents gave informed consent to participate in the study were first screened for HLA class II alleles conferring susceptibility to type 1 diabetes (HLA type was analysed from cord blood) and those with increased risk were recruited to the prospective follow-up study [15, 16]. A group of 64 children (34 boys) from the DIPP study whose parents consented to the OPV trial presented here were vaccinated using four serial doses of OPV (Polio Sabin, SB Biologicals, Rixensart, Belgium) at the age of 2, 3, 6 and 12 months (two drops in each oral dose). OPV included attenuated replication competent strains of the three poliovirus types (polioviruses 1,2 and 3). All these children carried $H L A-D Q B 1$ alleles conferring moderately increased risk for type 1 diabetes $(H L A-D Q B 1 * 0302 / x, x \neq D Q B 1 * 0201, * 0301$, $* 0602)$. A comparison group of 251 children received nonadjuvanted IPV [17] at their well baby clinic at the age of 6 and 12 months according to the standard national immunisation programme in Finland at that time (Fig. 1). All 64 children in the OPV group received a complete set of four serial vaccinations as planned, and there were no vaccine 


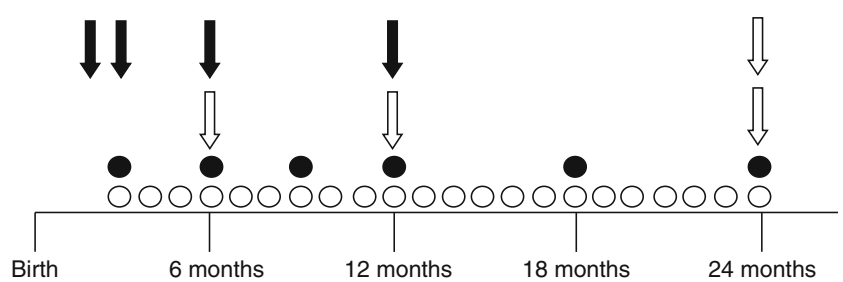

Fig. 1 Schematic presentation of the study protocol. Black and white arrows show the time of OPV and IPV administration, respectively. Black dots show the timing of the first six serum sample collections. White dots show the timing of stool sample collection every month from 3 to 24 months

refusals among the IPV group either. After the age of 12 months both groups were recommended to continue according to the national immunisation programme with IPV at the age of 2, 6 and 9-12 years. Both groups were recruited at the same DIPP study centre in the Tampere University Hospital during the same time period (1999-2000), thus they were all exposed to the same enterovirus seasons at the same age.

Islet autoantibody analyses All children were observed regularly from birth with blood samples taken at 3, 6, 9, 12, 18 and 24 months and at 12 month intervals thereafter for the analyses of islet autoantibodies. Islet autoantibodies were analysed from serum or plasma and included insulin autoantibodies (IAA), islet cell antibodies (ICA), insulinoma-associated protein 2 antibodies (IA-2A) and GAD antibodies (GADA) as previously described $[16,18]$. Positivity for multiple autoantibodies was defined as positivity for at least two of these autoantibodies during the whole follow-up period. Transient autoantibody positivity was defined as one or more positive samples, followed by at least two negative samples. Persistent autoantibody positivity was defined as at least two positive samples in a row, including the last sample obtained. Samples with maternal autoantibodies (antibodies detected initially in cord blood with decreasing levels in subsequent samples maximally up to the age of 15 months) were not included in the analyses. Clinical type 1 diabetes was diagnosed according to the ADA criteria.

Neutralising poliovirus antibodies Neutralising antibodies against poliovirus types 1-3 were analysed using the plaque assay to assess vaccine-induced immunity from plasma samples collected at 24 months from 64 children from each vaccine group (if a sample was not available at 24 months the previous sample was tested). The polioviruses (Sabin strains) were first incubated with $1 / 4$ or 1/64 dilution of plasma for $1 \mathrm{~h}$ at $36^{\circ} \mathrm{C}$ followed by an overnight incubation at room temperature using a method described previously [19]. The virus was then added on monolayers of Green Monkey kidney cells on six-well plates (Nunclon, NUNC, Denmark). The amount of infectious virus was measured by counting the plaques after $46 \mathrm{~h}$ of incubation at $36^{\circ} \mathrm{C}$. The plasma was taken as antibody positive if it blocked $>85 \%$ of the virus infectivity.
Detection of enterovirus RNA Stool samples were collected monthly at 3-24 months from all children in the OPV group and from 64 children in the IPV group. Thus, samples were taken sequentially at the same age forming a series of comparable samples in both groups. First a $10 \%$ stool suspension was prepared from the original stool sample in Hanks' solution including gentamycin, penicillin $\mathrm{G}$, amphotericin B and 4\% BSA. QIAamp viral RNA kit (Qiagen, Hilden, Germany) was used to extract the viral RNA. Stool samples were systematically screened for the presence of enterovirus and rhinovirus RNA using RT-PCR as previously described [20, 21]. This method amplifies a $115 \mathrm{bp}$ long fragment of 5'UTR of the viral genome which is common for all enteroviruses and rhinoviruses. The amplicons were linearly quantified with a liquid hybridisation assay using probes that detected either enterovirus or rhinovirus specific sequences. All positive samples were retested and all test runs included one virus positive control and two virus negative control samples. The analyses were carried out blind without knowing the vaccination history of the child, but the samples from the different vaccination groups were analysed in the same test runs.

Altogether 325 plasma samples from these children were analysed for the presence of enterovirus RNA using the same RT-PCR method (163 samples from the OPV group and 162 from the IPV group). These plasma samples were collected at 3-24 months of age and one to six samples were taken for each child (mean 2.5 samples per child in both groups). RNA extraction was performed from 500-1000 $\mu$ l of serum using the QIAamp UltraSens Virus Kit (Qiagen).

A restriction enzyme analysis was performed for PCR amplicons of all enterovirus-positive samples in order to distinguish OPV-derived poliovirus strains from non-polio enterovirus strains. At least one virus sequence of all available human enterovirus strains were obtained from GenBank for restriction site screening (218 strains in total). The specific restriction enzymes for Sabin strains were identified using the GeneDoc programme from the REBASE restriction enzyme collection $[22,23]$. The PCR amplicons of the Sabin 1 strain were cut with AvaI, Sabin 2 with AlwNI and Sabin 3 with PvuII in silico and laboratory tests. PCR amplicons of only one of the three tested Coxsackievirus A20 strains was cut with AlwNI, while none of the other non-polio enterovirus strains was cut with the selected poliovirus-specific enzymes. The restriction reactions were performed using $10 \mu \mathrm{l}$ of the PCR product according to manufacturer's protocol. The quantification of the uncut PCR product after restriction was performed with liquid phase hybridisation and the signal was compared with the signal of the original PCR product.

Statistical analyses The $\chi^{2}$ test and Fisher's exact test were used to analyse the statistical significance of differences observed between the children vaccinated with OPV and IPV. Kaplan-Meier survival analysis was carried out to assess the 
time to autoantibody positivity. The difference between follow-up times was analysed with the Mann-Whitney $U$ test. Cox regression was used to adjust for differences in number of siblings, pets in the family home and nursery/childcare attendance after 12 months of age between those receiving IPV vs OPV. The software packages used were SPSS version 22.0 (SPSS, Armonk, NY, USA) and Stata version 13.1 (StataCorp, College Station, TX, USA). Differences were considered significant at $p<0.05$.

Ethics The study was approved by the Pirkanmaa Hospital District Ethical Committee and the National Agency for Medicines in Finland (97193M KnipM, 98203M KnipM, clinicaltrials.gov: NCT02961595). Parents or guardians expressed their consent in writing.

\section{Results}

Both OPV and IPV induced clear immune responses to each of the three poliovirus types. All 64 children who received OPV developed neutralising antibodies to all the three poliovirus types ( $1 / 4$ titre). However, two of the 64 children in the IPV group who were tested for poliovirus antibodies remained negative for neutralising antibodies against poliovirus type 1 and one child remained negative for antibodies against poliovirus type 3 . The two children negative for poliovirus 1 lacked a plasma sample for 24 months and antibodies were analysed from earlier time points (9 months and 12 months) when they had received only one dose of IPV. The one child negative for poliovirus 3 was analysed at 24 months. High antibody titres (> 1/64) against all poliovirus types $1-3$ at 24 months were detected in $92 \%(46 / 50)$ of the children in the OPV group compared with $62 \%(31 / 50)$ of those in the IPV group $(p=0.001)$. The high antibody titres against poliovirus 1, 2 and 3 were observed in 96\%, 100\% and 96\% of the children vaccinated with OPV compared with $88 \%$ $(p=0.26), 90 \%(p=0.56)$ and $72 \%(p=0.002)$ of those vaccinated with IPV, respectively.

Enterovirus RNA was detected in $29.7 \%$ of the 744 stool samples collected from 3 to 24 months of age from children in the OPV group compared with $12.0 \%$ of the 744 stool samples from children in the IPV group $(p<0.001)$. This difference was seen in samples (470 in both groups) taken before the age of 12 months $(38.9 \%$ vs $11.3 \%$; $p<0.001)$ but not in later samples $(8.1 \%$ vs $7.7 \%)$. The restriction enzyme analyses confirmed the presence of Sabin poliovirus vaccine strains in $58.5 \%(107 / 183)$ of the enterovirus-positive samples in the OPV group, while no polioviruses were detected in the IPV group. Only one child in the OPV group did not have detectable enterovirus in stool samples but there were only three stool samples available from this child. Consequently, 98.4\% (63/64) of the children vaccinated with OPV had enterovirus-positive stool samples during the first year of life compared with $43.8 \%$ (28/64) of children vaccinated with IPV $(p<0.001)$. No difference was seen in rhinovirus positivity between the OPV and IPV groups $(9.9 \%$ vs $9.8 \%$ of stool samples were positive for rhinovirus RNA, respectively). Enterovirus RNA was detected in plasma in only three of the children in the OPV group and in three children in the IPV group (in $1.8 \%$ of the samples).

Altogether 4358 serum samples were analysed for diabetes-associated islet autoantibodies by December 2015 . The median follow-up time from birth to the last serum sample was 11.0 years and the total number of follow-up years was 3015.5 years. The follow-up time was at least 5 years in $75 \%$ of the OPV group and $68.9 \%$ of the IPV group. A follow-up time of 10 years and 15 years was completed in $50 \%$ and $30 \%$ of the children in the OPV group and in $52.6 \%$ and $25.9 \%$ of the children in the IPV group, respectively (electronic supplementary material [ESM] Fig. 1).

At least one autoantibody was detected at least once in 59 $(18.7 \%)$ children, with no difference between the groups (Table 1). This was true both among boys and girls. The first islet autoantibodies appeared at the median age of 6.1 years (range 0.7-11.9 years) in children who received OPV compared with 5.0 years (range $0.2-14.2$ years) in children who received IPV. Kaplan-Meier survival analysis did not show any difference in the time from birth to the first autoantibody positivity between the vaccination groups ( $p=0.616$, Fig. 2 ). The result remained the same after adjusting for sex, number of siblings, whether the household had pets and whether the child had started attending childcare/nursery before the age of 13 months. No difference was seen in the appearance of persistent islet autoantibodies ( $p=0.922$, ESM Fig. 2 ) or multiple autoantibodies between children vaccinated with IPV and OPV. Two or more different islet antibodies were observed in two children vaccinated with OPV (3.1\%, both boys) at the ages of 1.5 and 8.9 years. In the IPV group, multiple autoantibodies were detected in 17 children $(6.8 \%$, ten boys) at a median age of 4.9 years (range $0.6-12.0$ years) (Table 1 ). Persistently positive multiple islet antibodies were detected in only one $(1.5 \%)$ child vaccinated with OPV and eight (3.2\%) children vaccinated with IPV at a median age of 2.6 years (range 0.8-11.1 years). There was no difference between the vaccination groups (OPV vs IPV) in the frequency of the first appearing autoantibody as IAA ( $3.1 \%$ vs $4.4 \%)$, IA-2A (3.1\% vs $2.0 \%$ ) or GADA (1.6\% vs $2.4 \%)$. Maternal autoantibodies (antibodies in cord blood) were detected in two children, the follow-up samples were persistently negative in one child while the other developed ICA and IAA at 8 years of age.

Only one child (1.6\%) in the OPV group developed clinical type 1 diabetes at the age of 2.9 years. Four children (1.6\%) in the IPV group developed clinical diabetes at a mean age of 6.9 years (range 3.0-10.0 years). The children progressing to type 1 diabetes had been repeatedly positive for multiple islet autoantibodies. 
Table 1 Emergence of diabetesassociated islet autoantibodies in 64 children who received OPV and in 251 children who received IPV

\begin{tabular}{llll}
\hline & \multicolumn{2}{l}{ Type of vaccine } & \multirow{2}{*}{$p$ value } \\
\cline { 2 - 4 } & OPV & IPV & \\
& $n=64$ & $n=251$ & \\
\hline Boys, $n$ & 34 & 123 & \\
Median follow-up years from birth (range) & $10.9(1.0-15.2)$ & $11.1(0.5-15.9)$ & 0.969 \\
Median number of serum samples (range) & $15(4-24)$ & $15(2-38)$ & 0.990 \\
Median number of stool samples (range) & $11(2-20)$ & $11(2-20)$ & 1.000 \\
Enterovirus-positive stool samples, $n(\%)^{\mathrm{a}}$ & $221(29.7)$ & $89(12.0)$ & $<0.001$ \\
Seropositive for all polioviruses 1-3, $n(\%)^{\mathrm{b}}$ & $64(100)$ & $61(95.3)$ & 0.244 \\
Clinical type 1 diabetes, $n(\%)$ & $1(1.6)$ & $4(1.6)$ & 1.000 \\
At least one autoantibody ${ }^{\mathrm{c}}, n(\%)$ & $11(17.2)$ & $48(19.1)$ & 0.723 \\
Persistently positive autoantibodies ${ }^{\mathrm{d}}, n(\%)$ & $4(6.3)$ & $16(6.4)$ & 0.971 \\
Transiently positive autoantibodies ${ }^{\mathrm{e}}, n(\%)$ & $7(10.9)$ & $36(14.3)$ & 0.479 \\
At least two autoantibodies $(2-4), n(\%)$ & $2(3.1)$ & $17(6.8)$ & 0.384 \\
Two or three autoantibodies excluding ICA, $n(\%)$ & $1(1.6)$ & $10(4.0)$ & 0.701 \\
Individual autoantibodies, $n(\%)$ & & & \\
$\quad$ ICA & $10(15.6)$ & $48(19.1)$ & 0.519 \\
IAA & $2(3.1)$ & $14(5.6)$ & 0.541 \\
GADA & $1(1.6)$ & $12(4.8)$ & 0.479 \\
IA-2A & $2(3.1)$ & $9(3.6)$ & 1.000 \\
\hline
\end{tabular}

All children carry moderate HLA-conferred susceptibility to type 1 diabetes $(H L A-D Q B 1 * 0302 / x$, $x \neq D Q B 1 * 0201, * 0301, * 0602)$

${ }^{\text {a }}$ A total of 744 stool samples were collected from 64 children ( $n$, number of stool samples)

${ }^{\mathrm{b}}$ A subgroup of 64 children were tested in the IPV group

${ }^{\mathrm{c}}$ At least one autoantibody detected in any of the follow-up serum samples, maternal autoantibodies not included

${ }^{\mathrm{d}}$ At least two positive samples in a row, including the last sample obtained, implying that the last sample had to be positive

${ }^{\mathrm{e}}$ One or more positive samples, followed by at least two negative samples

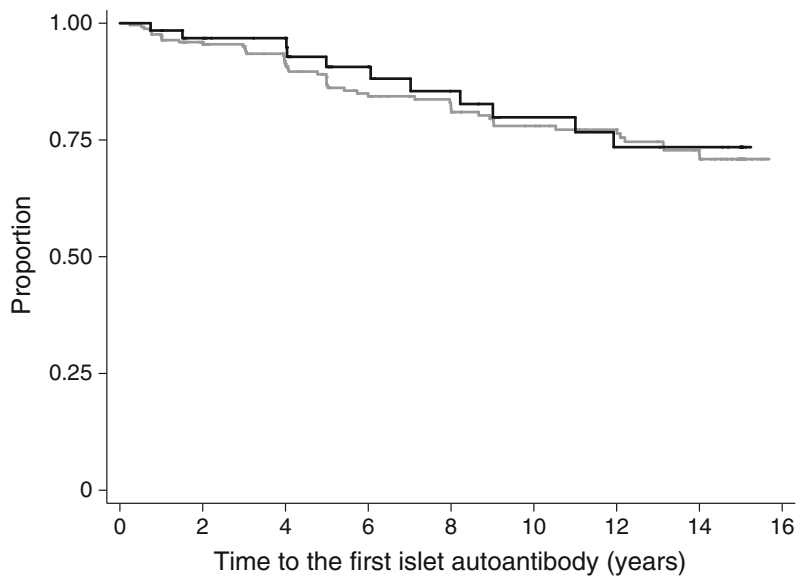

Fig. 2 Kaplan-Meier analysis of the time (years) from birth to the emergence of the first diabetes-associated islet autoantibody in 64 children who received OPV (black line) and in 251 children who received IPV (grey line). $p=0.616$

\section{Discussion}

Animal and human studies have shown that enterovirus infections may be involved in the pathogenesis of type 1 diabetes and a vaccine against potentially diabetogenic enterovirus strains would thus provide important information in the evaluation of causality. However, there have been concerns that immunisation against enteroviruses could induce islet autoimmunity and that vaccines could thus increase the risk of type 1 diabetes. For example, both inactivated and live attenuated vaccines could contain viral epitopes that are able to induce immunological cross-reactivity against beta cell proteins. Furthermore, live vaccines that cause an infection in vaccine recipients could induce beta cell damage by some additional mechanism. One such mechanism is related to the inflammation response that is induced by the replication of vaccine virus strains in the recipients. Live enterovirus vaccines that contain infectious viruses can lead to prolonged replication of vaccine virus strains in the intestinal mucosa and gut-associated lymphoid tissue. The close anatomical location of the pancreas and common lymphatic and vascular 
networks in this region may facilitate the spread of vaccineinduced inflammation from the intestine to the pancreas via soluble factors and activated lymphoid cells.

The results of the present study clearly show that sequential exposure to OPV significantly increased the detection of enterovirus RNA in stool samples. The rate of enterovirus positivity in stool samples in the IPV group was well in line with our earlier findings in Finnish children [24]. When compared with the IPV group, the children vaccinated with OPV had almost four times more enterovirus-positive stool samples at the age when OPV was administered ( $<1$ year of age) and this excess was due to increased detection of polioviruses. This indicates that OPV caused a marked increase in enterovirus exposure in the participating infants. Since these vaccine strains replicate in the gut administration also leads to inflammation and stimulates the gut-associated immune system that has been proposed to regulate the development of islet autoimmunity [25]. However, we did not find any evidence that this could have clearly increased or decreased the risk of islet autoimmunity. Our findings support earlier studies showing that poliovirus vaccines do not correlate with the appearance of islet autoantibodies or clinical type 1 diabetes [12-14]. In addition, this study shows that the type of vaccine (OPV or IPV) does not contribute to the risk. Moreover, the results are in line with epidemiological observations suggesting that type 1 diabetes incidence has mostly been lower in countries using OPV compared with countries using IPV [26, 27].

Both OPV and IPV induced neutralising antibodies but OPV induced stronger responses than IPV. This is logical since the children in the OPV group had received four vaccinations by the time neutralising antibodies were analysed compared with two vaccinations in the IPV group. The rate of enterovirus viremia was not higher in the OPV group compared with the IPV group. This probably reflects the attenuated nature of OPV vaccine virus strains as attenuated viruses replicate more slowly and cannot reach the blood during the first few days while the neutralising antibodies are not yet in place.

The children in the two study groups were purposefully very similar. The time of birth and geographical location were comparable between the groups; therefore, the children were exposed to the same enterovirus seasons and same kinds of enteroviruses circulating in their living environment. The children in both groups were also similar for their type 1 diabetesassociated HLA alleles that are known to affect the appearance of disease-associated autoantibodies [7]. Thus, it is unlikely that these factors could have biased the evaluation of association between OPV and IPV, and the risk of islet autoimmunity and type 1 diabetes. However, there are also some limitations in this study. First, this was not a randomised study but an open cohort study in a 'real-life setting'. In addition, the number of children receiving OPV was relatively small $(n=64)$ in line with the pilot-type design of this study. However, despite the relatively small number of children, the follow-up period was long (median 11.0 years). Altogether $88 \%$ of the children were observed for at least 2 years, which is the median age when multiple islet antibodies appeared in the Finnish children [5]. Moreover, $81 \%$ the of children were followed for at least 3 years, at which time, according to previous data from the DIPP study, $82 \%$ of children who later develop clinical type 1 diabetes already have seroconversion to positive islet autoantibodies [28]. The appearance of islet antibodies in the IPV group was similar to that previously seen in Finnish children with comparable HLA backgrounds [6]. However, it should be noted that the size of this study cohort limits the power to detect possible subtle differences between the groups.

In conclusion, this study suggests that the risk of islet autoimmunity does not differ between children vaccinated with OPV vs IPV. This indicates that the replication of attenuated enterovirus strains in the intestine does not increase the risk of type 1 diabetes.

Acknowledgements The staff of the Tampere DIPP clinic, the DIPP autoantibody and immunogenetics laboratories in Oulu and Turku, and the staff of the Department of Virology, University of Tampere are gratefully acknowledged for expert technical assistance.

Data availability The data that support the findings of this study are available on reasonable request from the author, HV.

Funding The recruitment of study participants and collection of stool samples in the DIPP study was funded by grants from JDRF, Sigrid Juselius Foundation, Reino Lahtikari Foundation, the Diabetes Research Foundation and the Academy of Finland. The oral polio vaccine (Polio Sabin) was provided by SB Biologicals, Rixensart, Belgium. The study sponsors were not involved in the design of the study; the collection, analysis, and interpretation of data; writing the report; or the decision to submit the report for publication

Duality of interest $\mathrm{HHy}$ and $\mathrm{MK}$ are minor shareholders $(<5 \%)$ in Vactech, a small biotech company developing picornavirus vaccines. There are no other potential conflicts of interest relevant to this article.

Contribution statement HV, SO, JI, RV, TV, JT, MK and HHy made substantial contributions to the study design, participated in the laboratory and carried out data analysis and interpretation. SH participated in the study design, and recruited and vaccinated the study population. $\mathrm{HHu}$ participated in the statistical analyses of the data. All authors helped to draft the manuscript, and read and approved the final version. HHy and MK are the guarantors of this work.

\section{References}

1. Morgan N, Richardson S (2014) Enteroviruses as causative agents in type 1 diabetes: loose ends or lost cause? Trends Endocrinol Metab 25:611-619

2. Hyöty H (2016) Viruses in type 1 diabetes. Pediatr Diabetes 17:56-64 
3. Hyöty H, Knip M (2014) Developing a vaccine for type 1 diabetes through targeting enteroviral infections. Expert Rev Vaccines 13: 989-999

4. Yi E-J, Kim J-H, Kim T-G, Chang S-Y (2017) Enterovirus 71 and vaccines. Clin Exp Vaccine Res 6:4-14

5. Ziegler AG, Rewers M, Simell O et al (2013) Seroconversion to multiple islet autoantibodies and risk of progression to diabetes in children. JAMA 309:2473-2479

6. Steck AK, Vehik K, Bonifacio E et al (2015) Predictors of progression from the appearance of islet autoantibodies to early childhood diabetes: the environmental determinants of diabetes in the young (TEDDY). Diabetes Care 38:808-813

7. Ilonen J, Kiviniemi M, Lempainen J et al (2016) Genetic susceptibility to type 1 diabetes in childhood - estimation of HLA class II associated disease risk and class II effect in various phases of islet autoimmunity. Pediatr Diabetes 17(Suppl 22):8-16

8. Whitton JL, Cornell CT, Feuer R (2005) Host and virus determinants of picornavirus pathogenesis and tropism. Nat Rev Microbiol 3:765-776

9. Pallansch M, Roos R (2007) Polioviruses, Coxsackieviruses, echoviruses and newer enteroviruses. In: Knipe DM, Howley PM (eds) Fields virology, 5th edn. Lippincott William \& Wilkins, Philadelphia, pp 878-884

10. Jenson AB, Rosenberg HS, Notkins AL (1980) Pancreatic islet-cell damage in children with fatal viral infections. Lancet 2:354-358

11. Richardson SJ, Willcox A, Bone AJ, Foulis AK, Morgan NG (2009) The prevalence of enteroviral capsid protein vp1 immunostaining in pancreatic islets in human type 1 diabetes. Diabetologia 52:1143-1151

12. Beyerlein A, Strobl A, Winkler C et al (2017) Vaccinations in early life are not associated with development of islet autoimmunity in type 1 diabetes high-risk children: results from prospective cohort data. Vaccine 35:1735-1741

13. EURODIAB Substudy 2 Study Group (2000) Infections and vaccinations as risk factors for childhood type I (insulin-dependent) diabetes mellitus: a multicentre case-control investigation. Diabetologia 43:47-53

14. Hviid A, Stellfeld M, Wohlfahrt J, Melbye M (2004) Childhood vaccination and type 1 diabetes. N Engl J Med 350:1398-1404

15. Seppälä E, Viskari H, Hoppu S et al (2011) Viral interference induced by live attenuated virus vaccine (OPV) can prevent otitis media. Vaccine 29:8615-8618
16. Kukko M, Kimpimäki T, Korhonen S et al (2005) Dynamics of diabetes-associated autoantibodies in young children with human leukocyte antigen- conferred risk of type 1 diabetes recruited from the general population. J Clin Endocrinol Metab 90:2712-2717

17. Hawken J, Troy SB (2012) Adjuvants and inactivated polio vaccine: a systematic review. Vaccine 19:6971-6979

18. Ilonen J, Hammais A, Laine AP et al (2013) Patterns of b-cell autoantibody appearance and genetic associations during the first years of life. Diabetes 62:3636-3640

19. Laitinen O, Honkanen H, Pakkanen O et al (2014) Coxsackievirus $\mathrm{B} 1$ is associated with induction of $\beta$-cell autoimmunity that portends to type 1 diabetes. Diabetes 63:446-455

20. Lönnrot M, Sjöroos M, Salminen K et al (1999) Diagnosis of enterovirus and rhinovirus infections by RT-PCR and time-resolved fluorometry with lanthanide chelate labeled probes. J Med Virol 59: 378-384

21. Oikarinen S, Tauriainen S, Viskari H et al (2009) PCR inhibition in stool samples in relation to age of infants. J Clin Virol 44:211-214

22. Nicholas KB, Nicholas HBJ (1997) GeneDoc: a tool for editing and annotating multiple sequence alignments. Distributed by the author. Available from www.psc.edu/biomed/genedocgenedoc. Accessed 1 May 2017

23. Roberts RJ, Vincze T, Posfai J, Macelis D (2015) REBASE - a database for DNA restriction and modification: enzymes, genes and genomes. Nucleic Acids Res 43:298-299

24. Honkanen H, Oikarinen S, Nurminen N (2017) Detection of enteroviruses in stools precedes islet autoimmunity by several months: possible evidence for slowly operating mechanisms in virusinduced autoimmunity. Diabetologia 60:424-431

25. Needell JC, Zipris D (2016) The role of the intestinal microbiome in type 1 diabetes pathogenesis. Curr Diab Rep 16:89

26. Atkinson MA, Eisenbarth GS, Michels AW (2014) Type 1 diabetes. Lancet 383:69-82

27. Sutter RW, Platt L, Mach O, Jafari H, Aylward RB (2014) The new polio eradication end game: rationale and supporting evidence. JID 210:S434-S438

28. Parikka V, Näntö-Salonen K, Saarinen M et al (2012) Early seroconversion and rapidly increasing autoantibody concentrations predict prepubertal manifestation of type 1 diabetes at genetic risk. Diabetologia 55:1926-1936 